\begin{tabular}{rllrrr} 
JD & \multicolumn{1}{c}{$195^{2}$} & & $V$ & $P$ & $P-V$ \\
2434259 & Sept. 4 & Maximum & $8^{\mathrm{m}} \cdot 33$ & $8^{\mathrm{m}} \cdot 95$ & $+\mathrm{O}^{\mathrm{m}} \cdot 63$ \\
289 & Oct. 4 & Minimum & 9.20 & 10.40 & $+\mathrm{I} .28$ \\
304 & Oct. 19 & Maximum & 8.32 & 9.28 & +0.99 \\
323 & Nov. 7 & Minimum & 8.78 & 9.92 & + I.17 \\
340 & Nov. 24 & Maximum & 8.34 & 9.23 & +0.90
\end{tabular}

The interval between the maxima before and after the deep minimum is 45 days, as compared with an interval of only 36 days between the maxima on either side of the shallow minimum. The star seems to be behind schedule by about I I days, as computed from the epoch and period given in the Kukarkin and Parenago General Catalogue of Variable Stars.

The unusual blueness of the maximum of Sept. 4 seems to have been caused almost entirely by excess light in the blue; the yellow maximum of that date was very similar in brightness to the other two which were observed.

This star is decidedly redder than the RV Tauri variables in globular clusters, as observed by Arp.

The differences between the blue and the yellow curves suggest that photoelectric observations of RV Tauri variables in several colors might well prove fruitful, especially if these observations could be combined with simultaneous spectrographic observations.

I. Ap. J. II4, I43, I95I.

Harvard College Observatory, Cambridge, Mass.

\section{Keenan, Philip C. Some clues to the luminosities of the S-type stars.}

About 70 stars brighter than the IIth visual magnitude can now be assigned to type $\mathrm{S}$. This material allows the following estimates of mean luminosities to be made.

I. Parallaxes from proper motions and radial velocities of long-period variables. The original solution by Merrill and Wilson in 1942 for I 7 stars gave $M_{\mathrm{v}} \approx-$ I.o. Two of their stars have now been eliminated from the class and two others have been added, but the solution is changed very little, giving $M_{\mathrm{v}} \approx-\mathrm{I} .5$.

2. Distances of variables of small amplitude lying in spiral arms of our galaxy. A plot of apparent magnitude against galactic longitude suggests that at least 6 of these stars having longitudes between $100^{\circ}$ and $160^{\circ}$ are situated at about the same distance from the sun in one of the spiral arms which have been observed in the direction of the anticenter. If they are in the arm approximately 2000 parsecs distant, which was found originally by Morgan, Sharpless and Osterbrock, and has been plotted also by Oort's group on the basis of their radio data, the luminosities lie in the range: $-2.5<M_{\mathrm{v}}<-4.5$.

An alternative possibility is that several of them are associated with some of the nearer stellar aggregates which Morgan has identified at distances of the order of 1000 parsecs in Camelopardus and Auriga. The S stars would then have absolute magnitudes nearer to -2 .

Further evidence will be needed to permit a decision between these alternatives. Acceptance of the lower luminosity would bring the group into closer agreement with the luminosities of the long-period variables, but on the other hand the greater galactic concentration of the variables of small amplitude would suggest that they might be more luminous than the long-period variables.

Perkins Observatory, Delaware, Ohio.

Kerr, F. J. and J. V. Hindman. Preliminary report on a survey of $2 \mathrm{I} \mathrm{cm}$ radiation from the Magellanic Clouds.

The Magellanic Clouds are sufficiently far away to be viewed as a whole, as isolated systems, and yet are sufficiently near for their internal structure to be discerned in some detail. A study of the $21 \mathrm{~cm}$ line radiation from neutral hydrogen within the Clouds has been made, using a 36-foot antenna mounted as a transit instrument. This paper gives a progress report on the analysis of the results.

The radiation was received from large areas of both Clouds, reaching beyond the optical limits. The observations, which were made with a resolution of one degree in angle, and $8 \mathrm{~km} / \mathrm{sec}$ in radial velocity, gave the line profile for about 250 independent points.

Optical observations have shown that the Large Cloud contains a substantial amount of obscuring dust, whereas the Small Cloud is essentially transparent. The Small Cloud was known to contain some gas, but the amount was thought to be much less than that in the Large Cloud.

The radio observations have shown however that the amount of neutral hydrogen is about the same in the two Clouds. Thus the ratio of gas to dust is very different in the two systems. 